\title{
Interrater Reliability in the Measurement of Flow Characteristics on Color-Coded Quantitative DSA of Brain

\author{
AVMs
}

(D).H Narsinh, (DK. Mueller, (D). Nelson, (D). Massachi, (DD.C. Murph, (D)A.Z. Copelan, (D).W. Hetts, (D) V.V. Halbach, (D) R.T. Higashida, (D)A.A. Abla, (D) M.R. Amans, DC.F. Dowd, (D) H. Kim, and DD.L. Cooke

\begin{abstract}
BACKGROUND AND PURPOSE: Hemodynamic features of brain AVMs may portend increased hemorrhage risk. Previous studies have suggested that MTT is shorter in ruptured AVMs as assessed on quantitative color-coded parametric DSA. This study assesses the interrater reliability of MTT measurements obtained using quantitative color-coded DSA.
\end{abstract}

MATERIALS AND METHODS: Thirty-five color-coded parametric DSA images of 34 brain AVMs were analyzed by 4 neuroradiologists with experience in interventional neuroradiology. Hemodynamic features assessed included MTT of the AVM and TTP of the dominant feeding artery and draining vein. Agreement among the 4 raters was assessed using the intraclass correlation coefficient.

RESULTS: The interrater reliability among the 4 raters was poor (intraclass correlation coefficient $=0.218 ; 95 \% \mathrm{Cl}, 0.062-0.414 ; P$ value $=.002$ ) as it related to MTT assessment. When the analysis was limited to cases in which the raters selected the same image to analyze and selected the same primary feeding artery and the same primary draining vein, interrater reliability improved to fair (intraclass correlation coefficient $=0.564 ; 95 \% \mathrm{Cl}, 0.367-0.717 ; P<.001$ ).

CONCLUSIONS: Interrater reliability in deriving color-coded parametric DSA measurements such as MTT is poor so minor differences among raters may result in a large variance in MTT and TTP results, partly due to the sensitivity and 2D nature of the technique. Reliability can be improved by defining a standard projection, feeding artery, and draining vein for analysis.

ABBREVIATIONS: $\mathrm{AUC}=$ area under the curve; bAVM = brain AVM; cDSA = color-coded parametric quantitative DSA; ICC = intraclass correlation coefficient; IQR = interquartile range; $\mathrm{PCA}=$ posterior cerebral artery; SCA = superior cerebellar artery

B rain AVMs (bAVMs) are uncommon high-flow vascular malformations that often present with intracranial hemorrhage, seizure, or headache in young adults. ${ }^{1-3}$ Increasingly, bAVMs are discovered incidentally during brain imaging performed for other reasons. ${ }^{4}$ However, management of bAVMs is challenging due to the risk of disability or death associated with hemorrhage, seizure, or infarct during the course of any of the common management strategies, which generally include observation, microsurgical resection, endovascular embolization, stereotactic radiosurgery, or

Received May 9, 2020; accepted after revision August 5.

From the Department of Radiology and Biomedical Imaging (K.H.N., J.M., D.C.M., A.Z.C., S.W.H., V.V.H., R.T.H., M.R.A., C.F.D., D.L.C.), Center for Cerebrovascular Research (J.N., H.K.), Department of Anesthesiology, and Department of Neurological Surgery (A.A.A.), University of California San Francisco, San Francisco, California; and Siemens Medical Solutions (K.M.), Malvern, Pennsylvania.

This work was supported by National Institutes of Health National Institute of Neurological Disorders and Stroke R01grant NS099628 and NIH NINDS U54 NS065705.

Please address correspondence to Daniel L. Cooke, MD, UCSF Department of Radiology \& Biomedical Imaging, 505 Parnassus Ave, L-349, San Francisco, CA, 94143; e-mail: Daniel.Cooke@ucsf.edu

- Indicates open access to non-subscribers at www.ajnr.org

http://dx.doi.org/10.3174/ajnr.A6846 a combination thereof. ${ }^{5}$ For example, in A Randomized Trial of Unruptured Brain AVMs (ARUBA), stroke or death occurred in $30.7 \%$ of patients in the interventional arm and $10.1 \%$ of patients in the medical management arm during a mean follow-up of 33.3 months. ${ }^{6}$ To better risk-stratify patients who would be best served by intervention versus medical management, improved imaging biomarkers are needed to determine which patients with unruptured bAVMs have a high risk of rupture and whether the administered treatments are effective in reducing this risk.

DSA remains the standard method for morphologic and hemodynamic characterization of bAVMs during endovascular interventions, as well as before and after radiosurgical or microsurgical treatment. Angioarchitectural features of bAVM morphology that portend an increased risk of hemorrhage include the presence of nidal or prenidal aneurysms, exclusive deep venous drainage, a single draining vein, venous outflow stenosis, or small nidus size. ${ }^{7-9}$ However, clinical and angioarchitectural features alone incompletely estimate hemorrhagic risk. ${ }^{10-13}$ For example, the $\mathrm{R}_{2} \mathrm{eD}$ AVM score has recently been proposed as a predictive tool to aid in hemorrhagic risk stratification, incorporating both clinical and angioarchitectural features. ${ }^{14}$ Specifically, 
the score is based on race, exclusive deep location, AVM size, exclusive deep venous drainage, and monoarterial feeding. However, the area under the curve (AUC) of the receiver operating characteristic of the $\mathrm{R}_{2} \mathrm{eD}$ AVM score is $0.685,{ }^{14}$ which may be characterized as "poor" or "fair" compared with other medical tests. Therefore, although the $\mathrm{R}_{2} \mathrm{eD}$ AVM score is an important advance in validating a hemorrhagic risk-prediction model, the incorporation of hemodynamic or genetic features may improve model performance and further validate its use. Specifically, hemodynamic risk factors such as a lower ratio of draining vein to feeding artery TTP, ${ }^{11,15}$ shorter MTT or TTP of the nidus, ${ }^{11,16}$ and lower mean total AVM flow, ${ }^{12}$ could be helpful in supplementing hemorrhagic risk assessment, ${ }^{13}$ but they cannot be easily measured during angiography.

Color-coded parametric quantitative DSA (cDSA) has been suggested as a surrogate marker of hemodynamics and a potential metric of hemorrhage risk ${ }^{11,13,17}$ and treatment effectiveness ${ }^{18}$ in bAVMs. cDSA converts a stack of 2D DSA images into a single composite parametric image that is color-coded according to the TTP opacification of contrast in each pixel. ${ }^{19}$ However, the reproducibility of these surrogate cDSA measurements of hemodynamics has not been assessed. In this study, we sought to assess the degree of interrater reliability in cDSA measurements among 4 neuroradiologists reviewing 35 cDSA images from 34 AVMs.

\section{MATERIALS AND METHODS}

\section{Patients}

Thirty-four consecutive patients with ruptured and unruptured bAVMs who were referred for cerebral angiography between June 2017 and December 2018 were included in the study. All patients provided written informed consent.

\section{Angiography and Data Postprocessing}

Using standard neuroangiographic techniques, we acquired DSA series on an Artis Q biplane angiography system (Siemens). A 5F catheter was advanced into the internal carotid or vertebral artery, and iodinated contrast was power-injected at a rate of 5$8 \mathrm{~mL} / \mathrm{s}$ for a total of $7-11 \mathrm{~mL}$, at a rate of 7.5 frames/s, depending on the feeding and draining vessels identified. All images from the angiographic study were reviewed by the study coordinator, and for each participant, an image with an appropriate projection to visualize the feeding and draining vessels separate from the nidus was designated for review by the raters.

Flow analysis software (syngo iFlow; Siemens) was used to create cDSA images. The diameter of a circular ROI was less than the caliber of a selected vessel, as independently drawn by each rater. In each case, ROIs of the same size were placed on the primary feeding artery and primary draining vein. ROIs were placed as close to the AVM nidus as possible with care to minimize overlap with other feeding arteries or draining veins. For each manually placed ROI, a time-versus-intensity graph was produced and exported by the software with calculated parameters of the following: 1) ROI peak time: time that the contrast intensity of a selected ROI reaches peak value; 2) ROI arrival time: time of arrival of contrast material; 3) MTT: average contrast material transit time through the target, measured as the time between the venous ROI peak and the arterial ROI peak; and 4) TTP: time elapsing from the first appearance of contrast material in the ROI to the peak contrast concentration in the ROI, that is, TTP $=$ ROI peak time - ROI arrival time. Additionally, the AUC of the timedensity graph was calculated as a surrogate marker of the total volume of blood passing through the ROI during the measured time period.

\section{Raters}

Four neuroradiologists (holding Certificates of Added Qualification in neuroradiology from the American Board of Radiology) specializing in interventional neuroradiology interpreted all cDSAs of bAVMs. Two interventional neuroradiology fellows participated, who were in their first and second years of their interventional neuroradiology fellowships and who had completed a diagnostic neuroradiology fellowship and at least 7 years of postgraduate training. Two interventional neuroradiology faculty participated, who had 8 and 35 years of interventional neuroradiology experience. Each rater was given short tutorials on drawing ROIs and a protocol instruction sheet to consult during their reads.

The tutorial and instruction sheet specified the goal of the task and the keystrokes required to open the prespecified images (selected by the study coordinator as described above), place ROIs, and store the quantitative data output. Raters were instructed to provide a qualitative assessment of the AVM flow rate (mild, moderate, or fast) relative to physiologic flow before beginning the postprocessing phase. Raters were then instructed to manually draw a circular ROI on the prespecified image and manually place it on the feeding artery as close to the nidus as possible while avoiding overlapping vasculature. After the initial ROI was placed, raters would make a copy of the circle (to ensure that the 2 were identical) and place it on the draining vein as close to the nidus as possible. Raters were permitted to consult with other neuroradiologists not involved in the study during interpretation.

\section{Statistical Analysis}

The intraclass correlation coefficient (ICC) was calculated to measure agreement of MTT among the 4 raters reviewing 35 AVM images from 34 different AVMs. The ICC calculations assumed a 2-way random-effects model (random raters and cases) with absolute agreement among raters. ICC calculations were also run on multiple subsets of the data on the basis of the following conditions: excluding instances when a rater did not use the prespecified image to review, excluding instances when a rater did not select the consensus feeding and draining vessels, and excluding highly influential outliers that exceeded the threshold of 4 SDs from the mean. Data analysis was conducted with STATA 15.1 (Release 15; StataCorp, 2017). ICC values were calculated using the user-created module KAPPAETC. ${ }^{20}$

\section{RESULTS}

Thirty-five AVM images from 34 AVMs were evaluated (Table 1). The median patient age was 43.5 years (interquartile range $[\mathrm{IQR}]=28.8-59.0$ years), and 14 (41\%) were women. Sixteen $(47 \%)$ AVMs were ruptured at diagnosis, 27 (79\%) were lobar, and 17 of 34 (50\%) had deep venous drainage. The median AVM size was $2.15 \mathrm{~cm}(\mathrm{IQR}=1.4-3.8 \mathrm{~cm})$.

The 35 images were each reviewed by the 4 raters (for a total of 140 reviews). In 135 (96\%) instances, the correct image (the 
Table 1: Patient and AVM characteristics

\begin{tabular}{lc}
\hline \multicolumn{1}{c}{ Characteristic } & Summary \\
\hline Count & 34 \\
Patient age (median) (IQR) (yr) & $43.5(28.8-59.0)$ \\
Female (No.) (\%) & $14(41)$ \\
AVM size (median) (IQR) (cm) & $2.2(1.4-3.8)$ \\
Ruptured prior imaging (No.) (\%) & $16(47)$ \\
Lobar location (No.) (\%) & $27(79)$ \\
Deep venous drainage (No./total) (\%) & $17 / 34(50)$ \\
\hline
\end{tabular}
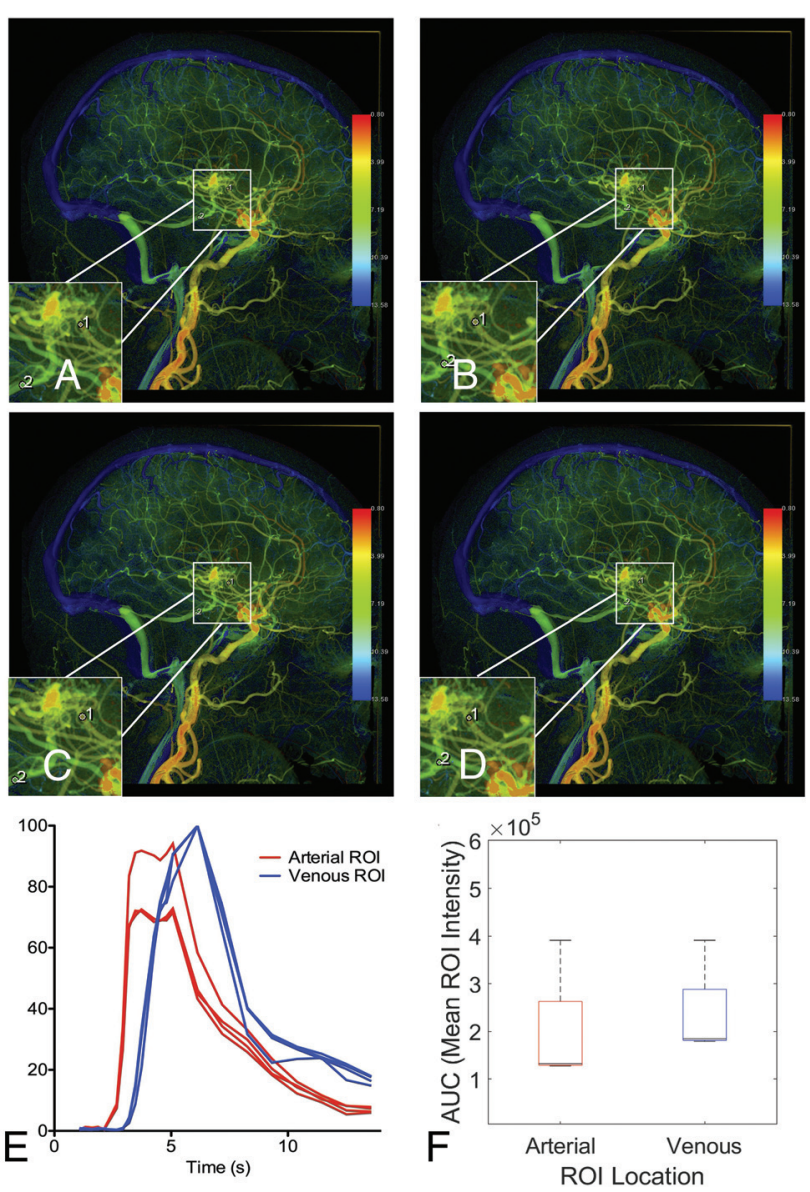

FIG 1. CDSA with low variance among raters in MTT results. Right internal carotid arteriogram in a lateral projection shows a right frontal operculum AVM supplied by 2 anterior cortical branches of the MCA, with dominant venous drainage into the vein of Labbe. All 4 raters $(A-D)$ chose the same image to interpret and placed ROls on the primary feeding artery and primary draining vein, in almost the same location. $E$, Time-density curves for the 4 raters are largely consistent, with the exception of a slightly larger arterial ROI by rater 3 (C) encompassing an adjacent overlapping vessel, which results in a larger AUC for the arterial ROI $(F)$. The peaks of the time-density curves are consistent, however, yielding reproducible results (median MTT of 1.73 seconds with $\mathrm{IQR}=1.06-2.4$ ).

image prespecified in the protocol sheet provided to the raters) was reviewed. When the correct image was reviewed, there were $116(86 \%)$ instances in which the consensus feeding artery was selected and 115 (85\%) instances in which the consensus draining vein was selected. In 100 (74\%) instances, both the consensus feeding and draining vessels were selected. The median MTT was 0.93 seconds ( $\mathrm{IQR}=0.54-1.20$ seconds), with a minimum value of -0.94 seconds (the only negative value) and a maximum of 7.06 seconds. Negative results for MTT do not have a physically interpretable meaning, due to overlapping vessels in the $2 \mathrm{D}$ projection resulting in an arterial ROI TTP greater than the venous ROI TTP.

Figure 1 demonstrates how the same cDSA image was interpreted by the 4 raters with the same vessels selected for analysis and low variance in cDSA results. On the lateral projection of a right internal carotid arteriogram, a right frontal operculum AVM is identified, supplied by 2 anterior cortical branches of the MCA, with dominant venous drainage into the vein of Labbe and minor venous drainage into the thalamostriate vein (not wellseen on CDSA). All 4 raters chose the correct image to interpret and chose the same feeding and draining vessels to analyze (ie, placed the ROIs on the same primary feeding artery and primary draining vein, though the ROI size slightly varied among raters). As a result, cDSA measurements were similar among raters, with a median MTT of 1.73 seconds (IQR $=1.06-2.4$ seconds).

Figure 2 demonstrates how the same cDSA image was interpreted by the 4 raters with high variance in results. On the lateral projection of a left vertebral arteriogram, an AVM in the dorsal vermis of the cerebellum is identified, with arterial supply from the left superior cerebellar artery (SCA) and left PICA and venous drainage into the vermian and tentorial veins. Both rater 1 (upper left) and rater 2 (upper right) placed ROIs on the same vessels (the SCA and tentorial vein) at nearly the same location, yet the MTT for rater 1 was 3.6 seconds, while the MTT for rater 2 was 0.7 seconds. The venous time-density curve for rater 1 has a larger second peak, which increased the calculated venous TTP. Rater 3 (lower left) selected an inferior vermian vein rather than a tentorial vein for the venous ROI placement. Rater 4 (lower right) selected the left PICA rather than the SCA for the arterial ROI placement. This case illustrates how raters differed in their assignment of the feeding and draining vessels, leading to cDSA measurements that were not reproducible, with a median MTT of 2.13 seconds ( $\mathrm{IQR}=0.50-4.66$ seconds).

Figure 3 demonstrates how the same cDSA image was interpreted by the 4 raters with different vessels selected for analysis but low variance in the cDSA results. On the lateral projection of a right vertebral arteriogram, a right occipital lobe AVM is identified, supplied by calcarine and parieto-occipital branches of the right posterior cerebral artery (PCA). Dominant venous drainage is into 2 internal occipital veins superior to the nidus, with minor drainage into a tentorial vein inferiorly. The tentorial venous egress overlaps the calcarine branch of the PCA feeding the AVM. All raters selected the calcarine branch of the PCA as the dominant arterial feeder but placed the ROI either proximal or distal to the overlapping tentorial venous egress. Three raters selected the more superior of the internal occipital veins as the primary draining vein, while 1 rater selected the smaller, more inferior of the internal occipital veins as the primary draining vein. For this participant, the median normalized linear distance between paired ROIs was $0.56(\mathrm{IQR}=0.41-0.85)$. As a result, the slopes and AUC of the arterial and venous time-density curves vary among raters. Nevertheless, the TTP measurements were similar among raters, with a median MTT of 0.53 seconds (IQR $=0.53-0.8$ seconds).

AJNR Am J Neuroradiol 41:2303-10 Dec 2020 www.ajnr.org 

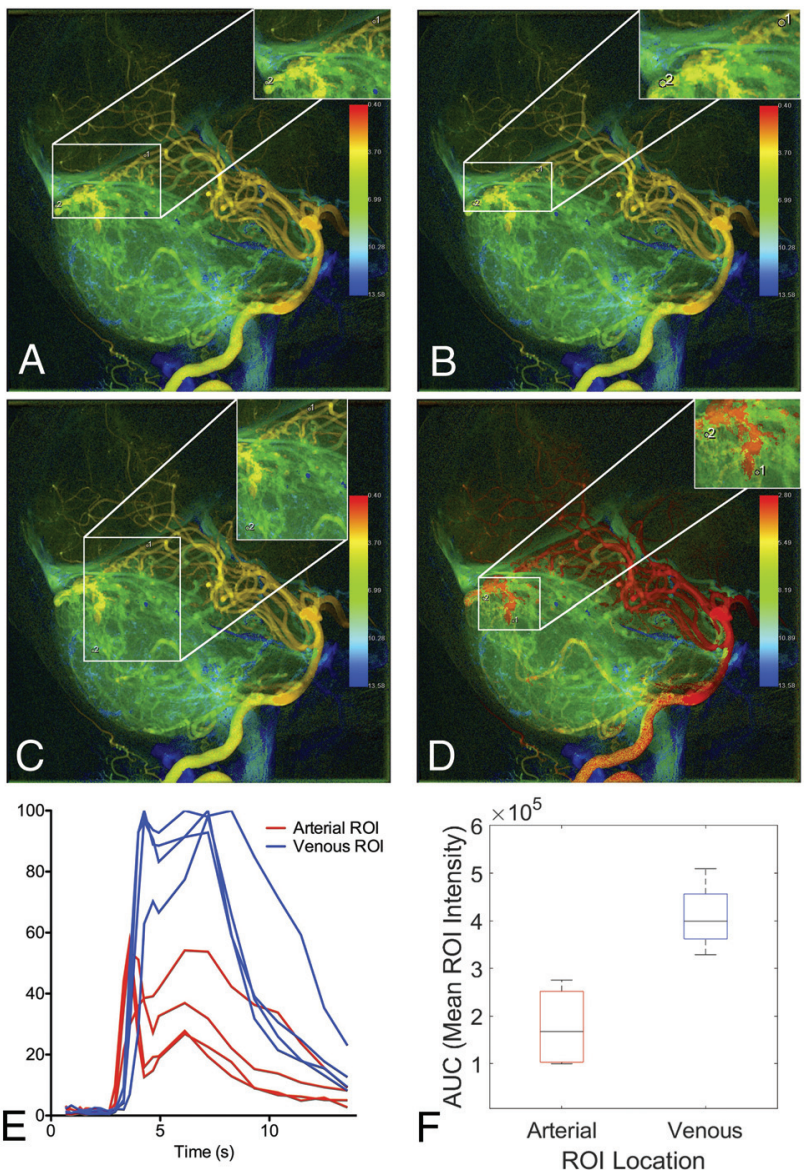

FIG 2. CDSA with high variance among raters in MTT results. Left vertebral arteriogram in a lateral projection shows a cerebellar AVM with arterial supply from the left SCA and left PICA and venous drainage into vermian and tentorial veins. $A$ and $B$, Raters 1 and 2 placed ROls on the same vessels, the SCA and tentorial vein, at nearly the same location, yet the MTT for rater $1(A)$ is 3.6 seconds, while the MTT for rater 2 is 0.7 seconds. The venous time-density curve for rater $1(A)$ has a larger second peak, which increased the calculated venous TTP. Rater $1(A)$ placed the $\mathrm{ROI}$ on a draining vein where it overlaps with a normal draining vein, and the second peak is a manifestation of the normal venous phase of the angiogram. $C$, Rater 3 selected an inferior vermian vein rather than a tentorial vein for venous $\mathrm{ROI}$ placement. $D$, Rater 4 selected the left PICA rather than the SCA for arterial ROI placement. Differences in ROI placement result in different time-density curves $(E)$ and calculated AUCs $(F)$.

Figure 4 is a scatterplot comparing MTT values of 2 of the 4 raters. The raters often disagreed on the feeding and draining vessels, placing ROIs on different feeding arteries and draining veins, and rarely used the wrong image for analysis (despite instructions to use a specified image).

We calculated that the ICC of the MTT among the 4 raters was 0.218 (95\% CI, 0.062-0.414; $P$ value $=.002$; Table 2$)$. When excluding instances when raters assessed the incorrect image, the ICC increased to 0.243 (95\% CI, 0.083-0.446; $P=.001)$. When we only considered ratings that chose the consensus feeding and draining vessels, the ICC increased to 0.564 (95\% CI, 0.3470.717; $P<.001)$. Results for the sensitivity analyses excluding outliers are presented in Table 2. Raters also made qualitative
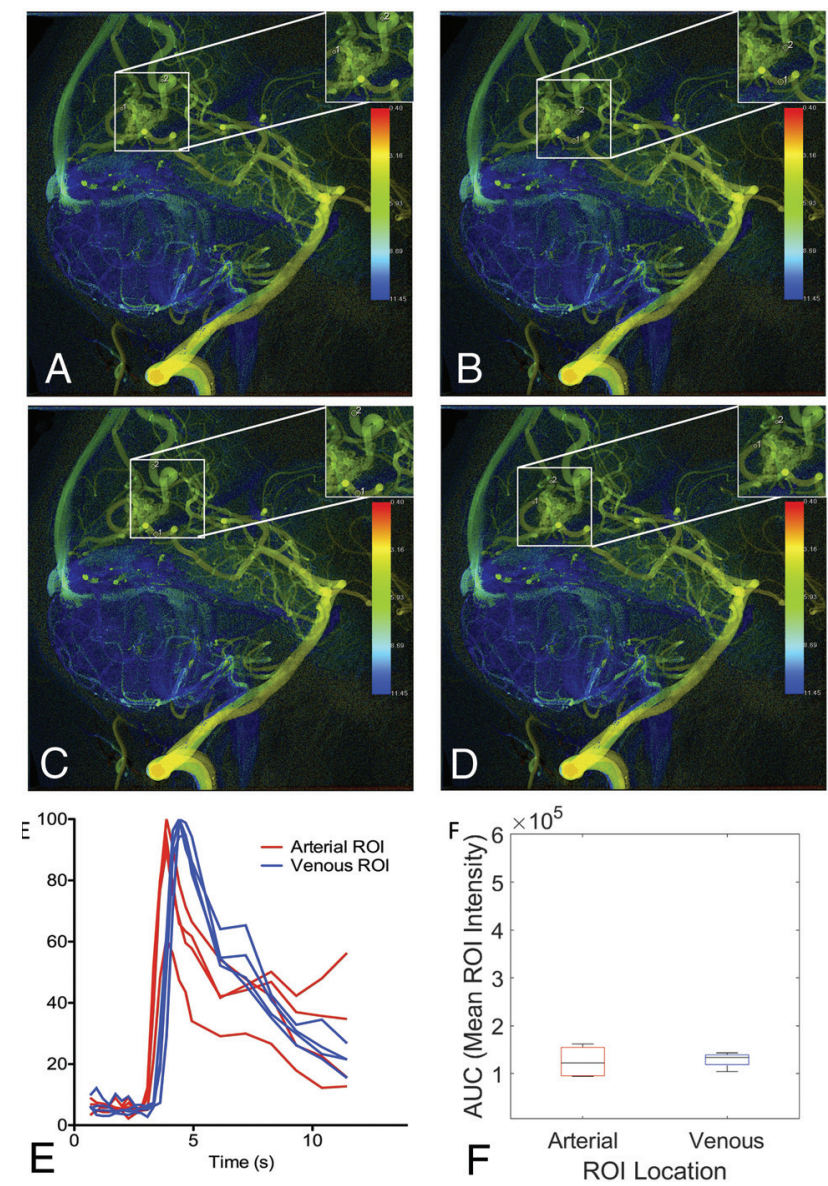

FIG 3. CDSA with variation among raters in vessels selected for analysis. Left vertebral arteriogram in a lateral projection shows a right occipital lobe AVM supplied by the calcarine and parieto-occipital branches of the right PCA, with dominant drainage into 2 internal occipital veins superior to the nidus and minor drainage into a tentorial vein inferiorly. A, Rater 1 placed the arterial $\mathrm{ROI}$ on the calcarine branch of the PCA distal to the overlapping minor venous egress and placed the venous ROI on the more superior internal occipital vein. $B$, Rater 2 placed the arterial ROI on the calcarine branch of the PCA proximal to the overlapping minor venous egress and placed the venous $\mathrm{ROI}$ on the more superior internal occipital vein. C, Rater 3 placed the arterial ROI on the calcarine branch of the PCA proximal to the overlapping minor venous egress and placed the venous ROI on the more superior internal occipital vein, though distal relative to rater 2. D, Rater 4 placed the arterial $\mathrm{ROI}$ on the calcarine branch of the PCA distal to the overlapping minor venous egress, similar to rater 1. Rater 4 also placed the venous $\mathrm{ROI}$ on the more inferior internal occipital vein, unlike the other raters. Despite differences in the vessels selected for analysis, time-density curves $(E)$ and AUCs $(F)$ were largely reproducible because the ROls were placed so close to the nidus by all raters.

assessments of flow (mild, moderate, or fast), which were in fair agreement ( $\kappa=0.33$; 95\% CI, $0.17-0.49 ; P<.001$ ).

We next sought to demonstrate how variability in ROI placement affects hemodynamic metrics. We selected 2 representative AVMs with a clearly identifiable feeding artery and draining vein and then placed 4 ROIs along the primary feeding artery and 4 ROIs along the primary draining vein to generate time-density curves and peak times. 
Figure 5 shows cDSA of a right internal carotid arteriogram in a lateral projection demonstrating a right frontal lobe AVM supplied by a frontopolar branch of the anterior cerebral artery with venous drainage into a frontal cortical vein that empties into the superior sagittal sinus. Arterial ROIs 1 and 3 include overlapping vessels that opacify in the normal arterial phase, resulting in longer peak times than arterial ROIs 2 and 4 . Venous ROI 2 includes an overlapping artery that opacifies in the normal arterial phase, resulting in a shorter peak time than venous ROIs 1, 3, and 4 .

Figure 6 show cDSA of a left internal carotid arteriogram in lateral projection, demonstrating a left parietal lobe AVM supplied by an enlarged callosomarginal branch of the left anterior cerebral artery, with venous drainage into a dilated left internal cerebral vein. Arterial ROI 1 is placed closest to the nidus, resulting in the largest peak time. Arterial ROI 3 is placed on a segment of the callosomarginal artery that courses laterally into the sulcus (labeled by an asterisk in lower left inset of Fig 5A), resulting in slightly increased contrast density within that ROI because of the in-plane course of that vessel segment and a smaller peak time (Fig 5D). Venous ROI 1 results in the largest AUC because it is placed closest to the nidus (Fig 6C), but the peak times are identical among venous ROIs 1, 2, and 4. However, venous ROI 3 results in a shorter peak time (Fig 6D) because of accumulation of contrast in that segment after ribbonlike streaming of contrast mixing with unopacified blood around the curve containing venous ROIs 1 and $2 .^{21}$

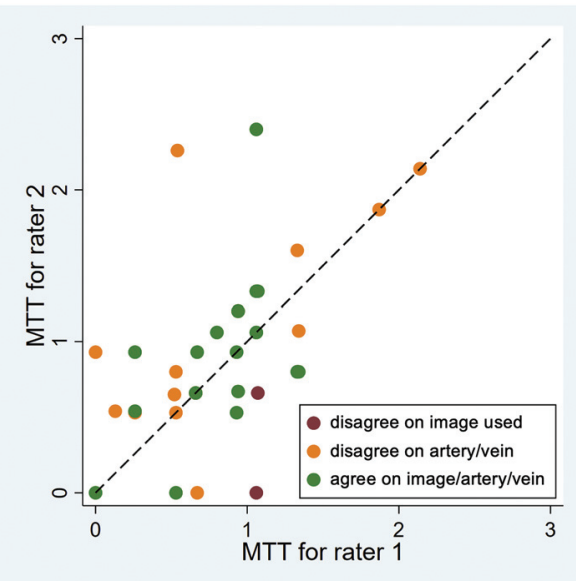

FIG 4. Interrater agreement between 2 representative raters. The calculated MTTs for raters 1 and 2 were compared (horizontal axis = rater 1 , vertical axis = rater 2). A dashed diagonal line represents perfect agreement between the 2 raters. When the same image, feeding artery, and draining vein are used for analysis, agreement improves. Similar interrater agreement was observed in other pair-wise comparisons of raters.

\section{DISCUSSION}

bAVMs are high-flow vascular malformations typified by transit of blood from arteries to veins through a nidus without a normal intervening capillary bed. The nidus typically has lower vascular resistance than normal capillaries; therefore, bAVMs are subject to a rapid rate of blood flow. These abnormal hemodynamics affect the molecular and structural composition of blood vessels in ways that are still being elucidated. For instance, in bulk RNA sequencing experiments comparing bAVMs with high and low flow, activation of Wnt signaling has been found as a feature of low-flow bAVMs. ${ }^{22}$ In vitro and murine in vivo models have found that connexin 37 expression is differentially regulated by shear stress, and that reduced expression of connexin 37 allows enlargement of capillaries and their conversion into arteriovenous shunts. ${ }^{23}$ Increased flow and resulting wall shear stress have also been implicated as factors associated with the growth of aneurysms, ${ }^{24-26}$ likely due to increased inflammation in the aneurysm wall. ${ }^{27}$ In addition to effects on the arteries, increased flow and pressure in the draining veins are associated with venous intimal hyperplasia that contributes to increased wall thickness and venous outflow stenosis, a known risk factor for bAVM hemorrhage. $^{12,28}$

Hemodynamic features are an important component of bAVM assessment in that one may gain insight into underlying pathobiology, individual hemorrhagic risk, and patient treatment goals. However, hemodynamic features of bAVMs are difficult to study because of complicated flow patterns through the nidus and the need for minimal invasiveness in assessing blood vessels at risk of rupture. Studies of bAVM hemodynamics have investigated the use of transcranial sonography, MRA, and DSA. An early study of transcranial sonography found no significant association with hemorrhage risk, ${ }^{29}$ but this technique has largely been supplanted by MRA due to its superior spatial coverage. The supposition that the increased flow rate in bAVMs contributes to prenidal and perinidal aneurysm formation is supported by the results of Shakur et al, ${ }^{30}$ who, using phase-contrast MRA, demonstrated increased wall shear stress in arterial afferents harboring aneurysms compared with arterial afferents that did not. On the other hand, Illies et $\mathrm{al}^{31}$ found that hemodynamic parameters on time-resolved MRA showed no association to known anatomic or angioarchitectural features of increased hemorrhage risk, including associated aneurysms. Rather, they found that MTT was increased in bAVMs that had previously ruptured, which did not seem to change or normalize with time, suggesting a permanent alteration in hemodynamics after rupture.

Catheter-based cerebral angiography remains the standard reference test for bAVMs because of its superior temporal and spatial resolution, allowing confident diagnosis typified by identification of an early draining vein relative to normal brain

Table 2: Intraclass correlation coefficients

\begin{tabular}{|c|c|c|c|c|c|c|c|c|}
\hline \multirow[b]{2}{*}{ Reviews Included } & \multicolumn{4}{|c|}{ Primary Analysis } & \multicolumn{4}{|c|}{ Sensitivity Analysis Excluding Outliers } \\
\hline & No. & ICC & $95 \% \mathrm{Cl}$ & $P$ Value & No. & ICC & $95 \% \mathrm{Cl}$ & $P$ Value \\
\hline All & 140 & 0.218 & $(0.062-0.414)$ & .002 & 138 & 0.463 & $(0.294-0.641)$ & $<.001$ \\
\hline Consensus images only & 135 & 0.243 & $(0.083-0.446)$ & .001 & 133 & 0.478 & $(0.307-0.655)$ & $<.001$ \\
\hline Consensus image, feeding artery, and draining vein & 100 & 0.564 & $(0.347-0.717)$ & $<.001$ & 100 & 0.564 & $(0.347-0.717)$ & $<.001$ \\
\hline
\end{tabular}

Note:-No. indicates total number of reviews included across all raters 

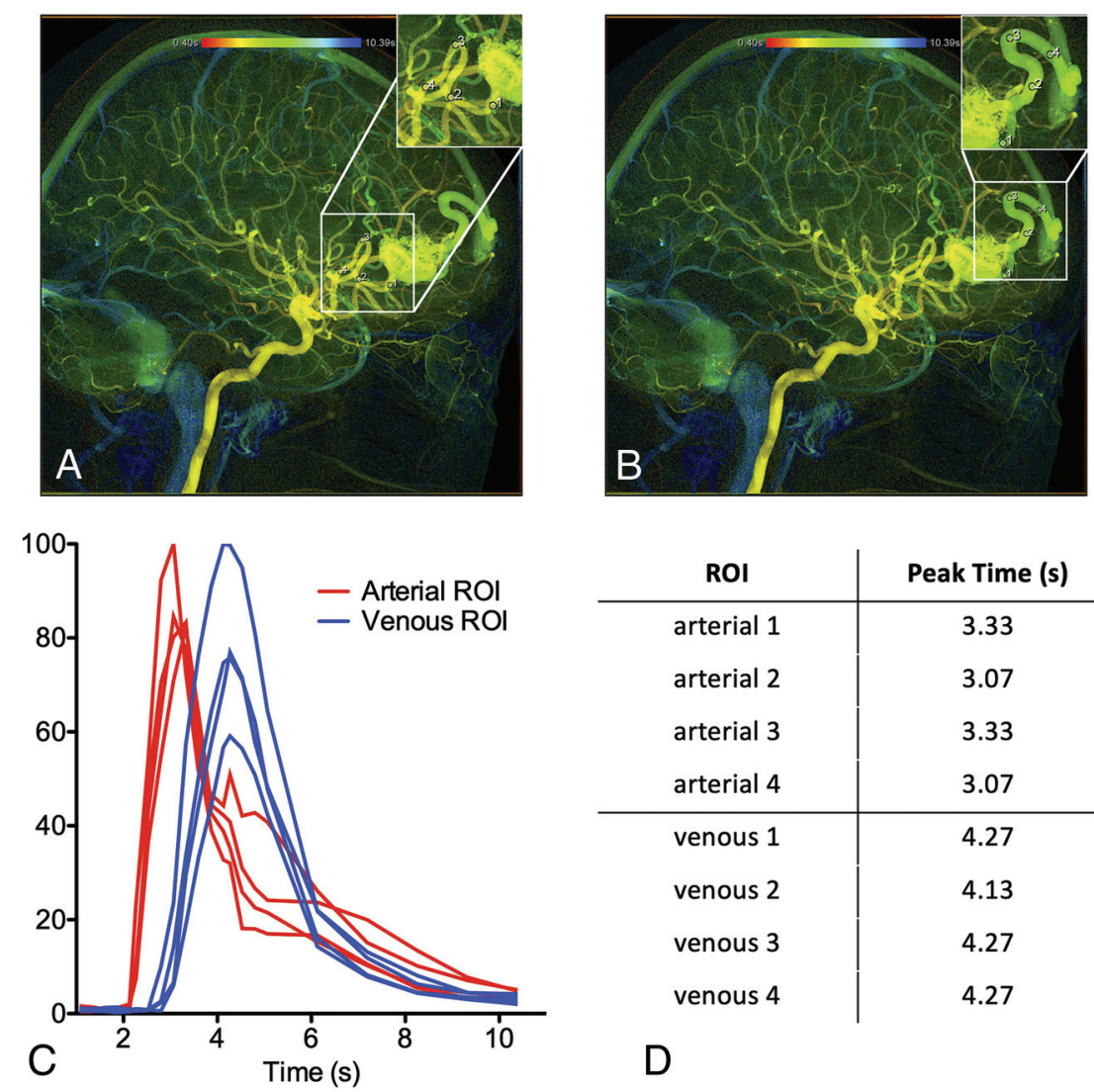

\begin{tabular}{c|c} 
ROI & Peak Time (s) \\
\hline arterial 1 & 3.33 \\
arterial 2 & 3.07 \\
arterial 3 & 3.33 \\
arterial 4 & 3.07 \\
\hline venous 1 & 4.27 \\
venous 2 & 4.13 \\
venous 3 & 4.27 \\
venous 4 & 4.27 \\
D
\end{tabular}

FIG 5. Variations in ROI placement can alter time-density curves and calculated peak times due to overlapping vessels. A right internal carotid arteriogram in a lateral projection demonstrates a right frontal lobe AVM supplied by the frontopolar branch of the right anterior cerebral artery with venous drainage into a frontal cortical vein, which drains into the superior sagittal sinus. $A$, Four arterial ROls were placed along the course of the arterial feeder with varying proximity to the nidus. $B$, Four venous ROIs were placed along the course of the draining vein with varying proximity to the nidus. $C$, The resulting time-density curves are not reproducible. $D$, The resulting peak times are not reproducible.

parenchyma, even if the nidus is small, and evaluation for angiographic features that inform hemorrhage risk and treatment risk. Thus, DSA is routinely performed to diagnose and assess angioarchitectural risk factors of bAVMs such as the presence of nidal or prenidal aneurysms, exclusive deep venous drainage, a single draining vein, venous outflow stenosis, or small nidus size. ${ }^{7-9}$ Hemodynamic metrics may augment hemorrhagic risk stratification models that are currently based on clinical and angioarchitectural features alone. ${ }^{11,16,17}$

cDSA can be obtained on the angiographic dataset using postprocessing software without additional contrast administration or an ionizing radiation dose. cDSA has been used by multiple groups to study hemodynamic parameters that may predict the natural history and/or treatment response of a particular AVM. ${ }^{11,16-18,32-34}$ However, the reproducibility of such measurements and operatordependence have not been evaluated. While angioarchitectural features have standardized terminology, ${ }^{35}$ no such standardization has yet been adopted for cDSA measurements.

Herein, we evaluated the interrater reliability among 4 neuroradiologists of cDSA measurements obtained during DSA of bAVMs. Among our group of raters, considerable variability was seen in the images and vessels used for cDSA analysis. Although reproducibility was fair in instances in which the same image and vessels were selected for analysis, ROI placement along the length of the vessel (ie, proximal to distal) varied among raters, leading to variability in results. Interrater reliability was poor overall but improved to fair in the subset of measurements in which raters agreed on the same image, feeding artery, and draining vein to analyze. Even when a consensus image, feeding artery, and draining vein were selected for analysis, differences in placement of the ROI (from proximal to distal) varied the impact of overlapping vessels on the resulting time-density curves and hence altered the derived metrics. When all raters placed ROIs close to the nidus without overlapping other vessels, results became more reproducible, though the finite temporal resolution of cDSA may preclude meaningful comparison of such results among patients. These results enjoin caution in the widespread application of cDSA without strict supervision of image analysis because measurements varied considerably among physicians in the current study, and variation among institutions has not yet been assessed. By comparison, inter- and intraobserver variability in the assessment of angioarchitectural features at high risk of hemorrhage, such as feeding artery aneurysms or intranidal aneurysms, is also poor. ${ }^{36}$

A $4 \mathrm{D}$ rotational flat panel CT could allow one to better select vessels for hemodynamic measurements in $3 \mathrm{D}$, thereby avoiding the challenge of overlapping vessel anatomy in the $2 \mathrm{D}$ projection space and possibly improving reproducibility. ${ }^{37,38}$ Such techniques require a separate rotational flat panel conebeam CT acquisition and are not based on the conventional 2D planar DSA images that serve as the current workhorse method for evaluating AVM angioarchitecture. When measuring hemodynamics using cDSA of 2D planar data, other groups have chosen to place ROIs at locations less susceptible to variation (eg, the cavernous internal artery or jugular bulb), which may improve reproducibility. However, if ROIs are placed at the skull base, a more general assessment of global hemispheric flow is obtained. Arteriovenous shunting of contrast due to the AVM is averaged with physiologic cerebral perfusion, a feature that may reduce noise in the calculation of MTT or other hemodynamic measures but may also reduce the magnitude of any meaningful hemodynamic change, thereby limiting the sensitivity of the method. In the future, reproducibility may be improved using postprocessing software with semiautomated ROI selection based on vessel-diameter 

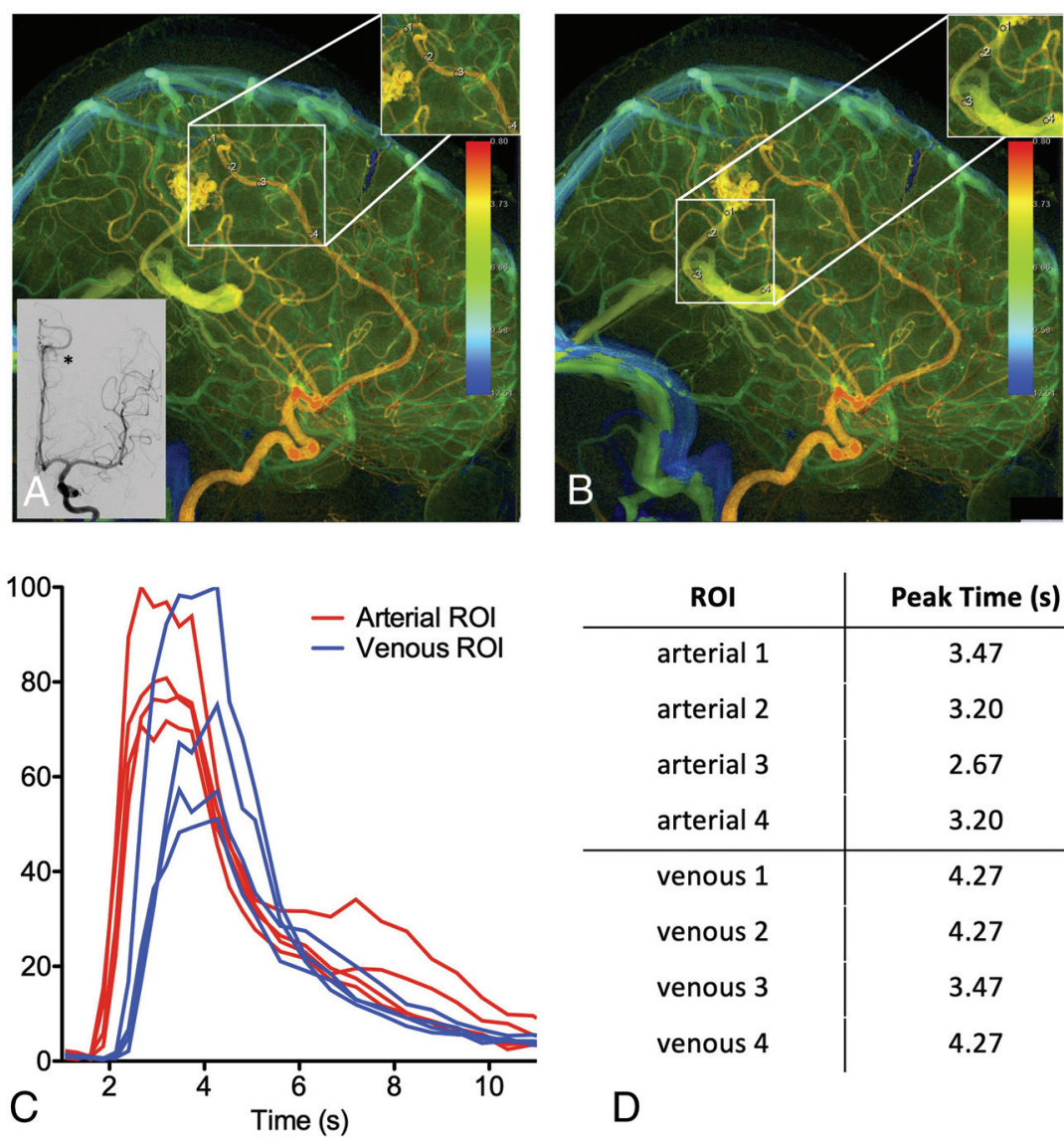

\begin{tabular}{c|c} 
ROI & Peak Time (s) \\
\hline arterial 1 & 3.47 \\
arterial 2 & 3.20 \\
arterial 3 & 2.67 \\
arterial 4 & 3.20 \\
\hline venous 1 & 4.27 \\
venous 2 & 4.27 \\
venous 3 & 3.47 \\
venous 4 & 4.27 \\
D
\end{tabular}

FIG 6. Variation in ROI placement can alter time-density curves and calculated peak times due to the in-plane vessel course and contrast mixing. Left internal carotid arteriogram in a lateral projection demonstrates a left parietal lobe AVM supplied by the left anterior cerebral artery with venous drainage into a dilated left internal cerebral vein. $A$, Four arterial ROls were placed along the course of the left anterior cerebral artery feeder with varying proximity to the nidus. $B$, Four venous ROls were placed along the course of the draining vein that empties into the left internal cerebral vein with varying proximity to the nidus. $C$, The resulting time-density curves are not reproducible. $D$, The resulting peak times are not reproducible.

thresholds. Automation in ROI placement based on computed juxtanidal arterial and venous diameters and avoidance of overlapping vessels would eliminate subjective assessments of primary afferent and efferent features.

\section{CONCLUSIONS}

Interrater reliability of cDSA measurements was poor overall but could be improved when raters agreed on the same image, primary draining artery, and primary draining vein to analyze. Caution should be used in interpretation of hemodynamic measures derived from cDSA because results may vary among physicians without strict supervision of image analysis.

Disclosures: Jeffrey Nelson—RELATED: Grant. National Institutes of Health.* Kerstin Mueller-RELATED: Other: employed by Siemens; UNRELATED: Employment: Siemens. Steven W. Hetts—UNRELATED: Grants/Grants Pending: Siemens, Comments: research contracts for X-ray angiography systems*. Christopher F. Dowd-UNRELATED: Other: Stryker, Comments: I serve as Chief Adjudicator of the Angio Core Lab for the EVOLVE flow-diverter clinical trial (department salary support).* Helen Kim—RELATED: Grant: National Institutes of Health, Comments: R01NS034949.* *Money paid to institution.

\section{REFERENCES}

1. Brown RD, Wiebers DO, Torner JC, et al. Frequency of intracranial hemorrhage as a presenting symptom and subtype analysis: a population-based study of intracranial vascular malformations in Olmsted County, Minnesota. J Neurosurg 1996;85:29-32 CrossRef Medline

2. Brown RD, Wiebers DO, Forbes G, et al. The natural history of unruptured intracranial arteriovenous malformations. J Neurosurg 1988;68:352-57 CrossRef Medline

3. Graf CJ, Perret GE, Torner JC. Bleeding from cerebral arteriovenous malformations as part of their natural history. J Neurosurg 1983;58:33137 CrossRef Medline

4. Morris Z, Whiteley WN, Longstreth $\mathrm{WT}$, et al. Incidental findings on brain magnetic resonance imaging: systematic review and meta-analysis. BMJ 2009;339:b3016 CrossRef Medline

5. Derdeyn CP, Zipfel GJ, Albuquerque FC, et al; American Heart Association Stroke Council. Management of Brain Arteriovenous Malformations: A Scientific Statement for Healthcare Professionals From the American Heart Association/American Stroke Association. Stroke 2017;48:e200-24 CrossRef Medline

6. Mohr JP, Parides MK, Stapf C, et al; International ARUBA investigators. Medical management with or without interventional therapy for unruptured brain arteriovenous malformations (ARUBA): a multicentre, non-blinded, randomised trial. Lancet 2014;383:614-21 CrossRef Medline

7. Alexander MD, Cooke DL, Nelson J, et al. Association between venous angioarchitectural features of sporadic brain arteriovenous malformations and intracranial hemorrhage. AJNR Am J Neuroradiol 2015;36:949-52 CrossRef Medline

8. Sahlein DH, Mora P, Becske T, et al. Features predictive of brain arteriovenous malformation hemorrhage. Stroke 2014;45:1964-70 CrossRef Medline

9. Stein K-P, Wanke I, Forsting M, et al. Associated aneurysms in supratentorial arteriovenous malformations: impact of aneurysm size on haemorrhage. Cerebrovasc Dis 2015;39:122-29 CrossRef Medline

10. Shakur SF, Liesse K, Amin-Hanjani S, et al. Relationship of cerebral arteriovenous malformation hemodynamics to clinical presentation, angioarchitectural features, and hemorrhage. Neurosurgery 2016;63:136-40 CrossRef Medline

11. Chen $X$, Cooke DL, Saloner D, et al. Higher flow is present in unruptured arteriovenous malformations with silent intralesional microhemorrhages. Stroke 2017;48:2881-84 CrossRef Medline

12. Shakur SF, Hussein AE, Amin-Hanjani S, et al. Cerebral arteriovenous malformation flow is associated with venous intimal hyperplasia. Stroke 2017;48:1088-91 CrossRef Medline

13. Norris JS, Valiante TA, Wallace MC, et al. A simple relationship between radiological arteriovenous malformation hemodynamics and clinical presentation: a prospective, blinded analysis of 31 cases. J Neurosurg 1999;90:673-79 CrossRef Medline 
14. Feghali J, Yang $\mathrm{W}, \mathrm{Xu}$, et al. R2eD AVM score. Stroke 2019;50:1703-10 CrossRef Medline

15. Raoult H, Bannier E, Maurel $\mathrm{P}$, et al. Hemodynamic quantification in brain arteriovenous malformations with time-resolved spin-labeled magnetic resonance angiography. Stroke 2014;45:2461-64 CrossRef Medline

16. Lin TM, Yang HC, Lee CC, et al. Stasis index from hemodynamic analysis using quantitative DSA correlates with hemorrhage of supratentorial arteriovenous malformation: a cross-sectional study. $J$ Neurosurg 2019 April 26. [Eub ahead of print] CrossRef Medline

17. Burkhardt JK, Chen X, Winkler EA, et al. Delayed venous drainage in ruptured arteriovenous malformations based on quantitative color-coded digital subtraction angiography. World Neurosurg 2017;104:619-27 CrossRef Medline

18. Rivera R, Sordo JG, Echeverria D, et al. Quantitative evaluation of arteriovenous malformation hemodynamic changes after endovascular treatment using parametric color coding: a case series study. Interv Neuroradiol 2017;23:650-55 CrossRef Medline

19. Strother CM, Bender F, Deuerling-Zheng Y, et al. Parametric color coding of digital subtraction angiography. AJNR Am J Neuroradiol 2010;31:919-24 CrossRef Medline

20. Klein DK. KAPPAETC: Stata Module to Evaluate Interrater Agreement. Boston College Department of Economics. 2019. https:// econpapers.repec.org/software/bocbocode/s458283.htm. Accesssd October 03, 2020.

21. Ruedinger KL, Harvey EC, Schafer S, et al. Optimizing the quality of 4D-DSA temporal information. AJNR Am J Neuroradiol 2019;40:2124-29 CrossRef Medline

22. Huo $\mathrm{R}, \mathrm{Fu} \mathrm{W}, \mathrm{Li} \mathrm{H}$, et al. RNA sequencing reveals the activation of Wnt signaling in low flow rate brain arteriovenous malformations. J Am Heart Assoc 2019;8:e012746 CrossRef Medline

23. Peacock HM, Tabibian A, Criem N, et al. Impaired SMAD1/5 mechanotransduction and $\mathrm{Cx} 37$ (connexin 37) expression enable pathological vessel enlargement and shunting. Arterioscler Thromb Vasc Biol 2020;40:e87-104 CrossRef Medline

24. Cebral JR, Mut F, Weir J, et al. Association of hemodynamic characteristics and cerebral aneurysm rupture. AJNR Am J Neuroradiol 2011;32:264-70 CrossRef Medline

25. Sforza DM, Kono K, Tateshima S, et al. Hemodynamics in growing and stable cerebral aneurysms. J Neurointerv Surg 2016;8:407-12 CrossRef Medline

26. Varble N, Rajabzadeh-Oghaz H, Wang J, et al. Differences in morphologic and hemodynamic characteristics for "PHASES-based" intracranial aneurysm locations. AJNR Am J Neuroradiol 2017;38:2105-10 CrossRef Medline

27. Cebral J, Ollikainen E, Chung BJ, et al. Flow conditions in the intracranial aneurysm lumen are associated with inflammation and degenerative changes of the aneurysm wall. AJNR Am J Neuroradiol 2017;38:119-26 CrossRef Medline

28. Viñuela F, Nombela L, Roach MR, et al. Stenotic and occlusive disease of the venous drainage system of deep brain AVM's. J Neurosurg 1985;63:180-84 CrossRef Medline

29. Kader A, Young WL, Pile-Spellman J, et al. The influence of hemodynamic and anatomic factors on hemorrhage from cerebral arteriovenous malformations. Neurosurgery 1994;34:801-08 CrossRef Medline

30. Shakur SF, Amin-Hanjani S, Mostafa H, et al. Hemodynamic characteristics of cerebral arteriovenous malformation feeder vessels with and without aneurysms. Stroke 2015;46:1997-99 CrossRef Medline

31. Illies T, Forkert ND, Saering D, et al. Persistent hemodynamic changes in ruptured brain arteriovenous malformations. Stroke 2012;43:2910-15 CrossRef Medline

32. Todaka T, Hamada JI, Kai Y, et al. Analysis of mean transit time of contrast medium in ruptured and unruptured arteriovenous malformations. Stroke 2003;34:2410-14 CrossRef Medline

33. Shellikeri S, Bai H, Setser RM, et al. Association of intracranial arteriovenous malformation embolization with more rapid rate of perfusion in the peri-nidal region on color-coded quantitative digital subtraction angiography. J Neurointerv Surg 2020;12:902-05 CrossRefMedline

34. Ma GM, Dmytriw AA, Patel PA, et al. Quantitative color-coded digital subtraction neuroangiography for pediatric arteriovenous shunting lesions. Childs Nerv Syst 2019;35:2399-403 CrossRef Medline

35. Atkinson RP, Awad IA, Batjer HH, et al; Joint Writing Group of the Technology Assessment Committee American Society of Interventional and Therapeutic Neuroradiology; Joint Section on Cerebrovascular Neurosurgery a Section of the American Association of Neurological Surgeons and Congress of Neurological Surgeons; Section of Stroke and the Section of Interventional Neurology of the American Academy of Neurology. Reporting terminology for brain arteriovenous malformation clinical and radiographic features for use in clinical trials. Stroke 2001;32:1430-42 CrossRef Medline

36. Iancu-Gontard D, Weill A, Guilbert F, et al. Inter- and intraobserver variability in the assessment of brain arteriovenous malformation angioarchitecture and endovascular treatment results. AJNR Am J Neuroradiol 2007;28:524-27 Medline

37. Lin EY, Lee RC, Guo WY, et al. Three-dimensional quantitative color-coding analysis of hepatic arterial flow change during chemoembolization of hepatocellular carcinoma. J Vasc Interv Radiol 2018;29:1362-68 CrossRef Medline

38. Meijs M, Pegge S. A H, Murayama K, et al. Color-mapping of 4DCTA for the detection of cranial arteriovenous shunts. AJNR Am J Neuroradiol 2019;40:1498-1504 CrossRef Medline 